\title{
POSITIVE BASES FOR LINEAR SPACES $(1,2)$
}

\author{
BY \\ RICHARD L. MCKINNEY
}

INTRODUCTION

Motivated by developments in the theories of games and linear programming, Davis [2] introduced the concepts of positively spanning sets and positive bases in Euclidean spaces. These are analogous to the ordinary notions of linear spanning sets and linear bases. We here continue the investigation initiated by Davis, our attention being directed especially to the infinitedimensional theory and its contacts with the theory of convex sets in linear spaces.

We begin by considering various characterizations of positively spanning sets. Some of these will be of a geometric nature, and lead to consideration of the semi-spaces first defined by Hammer [3]. $\$ 2$ is concerned with positive bases and their dependence on certain minimal subspaces. Generalizing a method of Davis, we are able in $\S 3$ to characterize positive bases as linearly spanning sets which admit a certain type of real function. Finally, in $\S 4$, we show that even though an arbitrary positive basis may lack certain desirable properties possessed by linear bases, there are special classes of positive bases which do have some of these properties.

Throughout, we use the symbols $\cup, \cap$, and $\sim$ for set union, intersection, and difference respectively. If $A$ and $B$ are subsets of a linear space then $A+B=\{a+b \mid a \in A, b \in B\}$ and $-A=\{-a \mid a \in A\}$. The cardinality of a set $A$ is denoted by card $A$. $L$ will denote an arbitrary real linear space. Elements of $L$ will be represented by lower case letters, excluding $r, s$ and $t$, which will usually represent real numbers, and $i, j, k, l, m$ and $n$, which will denote integers. The neutral element of $L$ will be denoted by $\varnothing$, the empty set by $\Lambda$, and real-valued functions by Greek letters.

\section{Positively Spanning Sets and Semispaces}

1.1. Introduction. For a subset $A$ of $L$, let $\& A$ be the set of all real valued functions on $A$ which vanish (i.e., have value 0 ) at all but a finite number of points of $A$. Denote by $\mathfrak{P} A$ the set of all non-negative members of $\mathbb{R} A$, i.e., those members of $\& A$ which are non-negative at each point of $A$. For $\lambda \in \mathbb{R} A$, let $\lambda_{a}$ be the value of $\lambda$ at $a$. For $\lambda \in R A$, we shall say that a appears in $\lambda$ if and only if $\lambda_{a} \neq 0$. If $a$ appears in $\lambda$ we shall also say that $\lambda$ involves $a$. A linear

Presented to the Society, November 22, 1958; received by the editors July 27, 1960 and, in revised form, June 13, 1961.

(1) This paper constitutes part of a thesis submitted to the University of Washington. The author would like to thank V. L. Klee, Jr. for supervising the research.

(2) Sponsored in part by the Office of Ordnance Research, U. S. Army. 
combination of $A$ is a point of the form $\sum_{a \in A} \lambda_{a} a$ for $\lambda \in R A$. A positive combination of $A$ is a point of the form $\sum_{a \in A} \lambda_{a} a$ for $\lambda \in \Re A$. We define lin $A$ and pos $A$ to be (respectively) the set of all linear combinations of $A$ and the set of all positive combinations of $A$. A linear relation over $A$ is a member $\lambda$ of $\mathfrak{\&} A$ such that $\sum_{a \in A} \lambda_{a} a=\varnothing$. Similarly, a positive relation over $A$ is a member $\lambda$ of $\mathfrak{B} A$ such that $\sum_{a \in A} \lambda_{a} a=\varnothing$. A set $A \subset L$ linearly spans $L$ if and only if lin $A=L$, and positively spans $L$ if and only if pos $A=L$.

1.2. Elementary properties of positively spanning sets. The following properties of positively spanning sets follow immediately from the definitions involved.

1. If $B$ is a subset of $L$ which linearly spans $L$ then the set $B \cup(-B)$ positively spans $L$.

2. If a set $A$ positively spans $L$ then $A$ linearly spans $L$.

3. If a set $B$ contains a subset $A$ which positively spans $L$ then $B$ positively spans $L$.

4. If $A$ is a subset of $L$ then $\operatorname{lin} A$ is a linear subspace of $L$ and pos $A$ is a convex cone with vertex $\varnothing$ in $L$. (See $\$ 1.3$ for the definitions involved.)

Since the converse of 2 above is clearly false, it follows that the hypothesis of positive spanning is in general stronger than that of linear spanning. A useful characterization of this strengthening is given by Theorem 1.1 and Corollary 1.2 which are essentially Theorems 3.6 and 3.7 of Davis [2]. We include the proofs here for the sake of completeness.

ThEorem 1.1. Suppose $A \subset L \sim\{\varnothing\}$ linearly spans $L$. Then the following are equivalent:

(1) A positively spans $L$.

(2) For each $a \in A,-a \in \operatorname{pos}(A \sim\{a\})$.

(3) Every $a \in A$ appears in some positive relation over $A$.

Proof. To prove (1) implies (2) let $a \in A$. Then we have (assuming $A$ positively spans $L):-a=\sum_{b \in \Delta} \lambda_{b} b$ where $\lambda \in \mathfrak{P} A$. If we define $\gamma \in \mathfrak{B}(A \sim\{a\})$ by the equations

$$
\gamma_{c}=\frac{\lambda_{c}}{1+\lambda_{a}} \quad \text { for } c \in(A \sim\{a\})
$$

then

$$
\sum_{c \in(A \sim\{a\})} \gamma_{c} c=\sum_{c \in(A \sim\{a\})} \frac{\lambda_{c}}{1+\lambda_{a}} c=\frac{1}{1+\lambda_{a}}\left(-a-\lambda_{a} a\right)=-a .
$$

If (2) holds we have $-a=\sum_{b \in(A \sim\{a\})} \lambda_{b} b$ for some $\lambda \in \mathfrak{B}(A \sim\{a\})$. But then $a$ appears in the relation $\beta \in \mathfrak{B} A$ defined by $\beta_{c}=\lambda_{c}$ for $c \neq a$ and $\beta_{a}=1$. Thus (2) implies (3).

Now to show (3) implies (1), let $d$ be an arbitrary element of $L$. Then since $A$ linearly spans $L$ we have $d=\sum_{a \in A} \lambda_{a} a$ for some $\lambda \in \mathcal{R} A$. Now let 
$B=\left\{a \mid a \in A\right.$ and $\left.\lambda_{a}<0\right\}$. By (3), for each $b \in B$ there exists an $\alpha^{b} \in \Re A$ such that $\sum_{a \in A} \alpha_{a}^{b} a=\varnothing$ and $\alpha_{b}^{b}>0$; we may clearly pick $\alpha^{b}$ so that $\left.\alpha_{b}^{b}\right\rangle\left|\lambda_{b}\right|$. Now let $\delta=\left(\sum_{b \in B} \alpha^{b}\right)+\lambda$. Then $\delta \in \mathfrak{B} A$ and

$$
d=\sum_{a \in A} \delta_{a} a
$$

Hence, $A$ positively spans $L$.

Corollary 1.2. If $A$ positively spans a linear space $L$ then $A \sim\{a\}$ linearly spans $L$ where $a$ is an arbitrary member of $A$.

Proof. This is an immediate consequence of (2) in Theorem 1.1.

Following the usual definitions, we will say that a subset $B$ of a linear space $L$ is linearly dependent if and only if there exists a linear relation $\lambda$ over $B$ in which some member of $B$ appears. A subset of a linear space which is not linearly dependent is linearly independent. An equivalent definition is, of course, to call a set $B$ linearly independent if and only if no member $b \in B$ is a linear combination of $B \sim\{b\}$. In $\S 2$ we shall see that it is the latter definition which provides the analogy for defining positive independence.

Corollary 1.2 is the best possible result in the sense that it is not always possible to remove two elements from a set which positively spans $L$ and still have $L$ linearly spanned by those remaining. In fact, if $L$ is an arbitrary linear space let $B$ be a linear basis for $L$. Then $A=B \cup(-B)$ positively spans $L$ but if $a$ is an arbitrary element of $A$ the set $A \sim\{a,-a\}$ does not linearly span $L$.

If $A$ positively spans a finite-dimensional $L$ then it may not be possible to find any two elements $a_{1}$ and $a_{2}$ of $A$ such that $A \sim\left\{a_{1}, a_{2}\right\}$ linearly spans $L$. This will follow from the fact (see 2.3) that in each finite-dimensional linear space $L$ there is a set $A$ which positively spans $L$ and such that $A \sim\{a\}$ is a linear basis, a fortiori is linearly independent, for an arbitrary $a \in A$. The corollaries of the following theorem show that the situation is different for an infinite-dimensional $L$.

TheOREm 1.3. Let $X$ and $Y$ be subsets of a linear space L. If $X$ is infinite and linearly independent, and card $Y<\operatorname{card} X$, then $-X \mp \operatorname{pos}(X \cup Y)$, (and hence pos $(X \cup Y)$ is not a subspace).

Proof. We may assume $X \cap Y=\Lambda$, since the theorem is equivalent to that with $Y$ replaced by $Y^{\prime}=Y \sim(X \cap Y)$. Suppose the conclusion of the theorem is false, i.e., for each $x \in X$ there exists a $\lambda^{x} \in \mathfrak{B}(X \cup Y)$ such that $-x$ $=\sum_{z \in X \cup Y} \lambda_{z}^{x} z$. Now define $F_{x}=\left\{y \mid y \in Y\right.$ and $\left.\lambda_{y}^{x} \neq 0\right\}$ for each $x \in X$. Since for each $x \in X, F_{x}$ is a finite subset of $Y$ we have card $\left\{F_{x} \mid x \in X\right\} \leqq$ card $Y$ if card $Y$ is infinite. Since $X$ is infinite and card $Y<$ card $X$ it follows that card $\left\{F_{x} \mid x \in X\right\}<\operatorname{card} X$ whether card $Y$ is infinite or not. Hence there must exist a finite $G \subset Y$ and an infinite $H \subset X$ such that $F_{x}=G$ for all $x \in H$. Let $n$ be the number of elements of $G$. For each $x \in H$ consider the positive relation 
$\gamma^{x}$ over $X \cup Y$ defined by the condition $\gamma_{z}^{x}=\lambda_{z}^{x}$ for $z \neq x$ and $\gamma_{z}^{x}=\lambda_{x}^{x}+1$ for $z=x$. From $\left\{\gamma^{x} \mid x \in H\right\}$ we can pick an ordered set

$$
C_{0}=\left\{\gamma^{x_{1}}, \gamma^{x_{2}}, \cdots, \gamma^{x_{2 n}}\right\}
$$

of $2^{n}$ positive relations such that for each $i, 1 \leqq i \leqq 2^{n}$, there exists in $X$ an $x_{i}^{\prime}$ appearing in $\gamma^{x_{i}}$ but not in $\gamma^{x_{k}}$ for $k<i$. Consider each pair $\left(\gamma^{x_{i}}, \gamma^{x_{i+1}}\right)$ for $i$ odd. Eliminating an element $g_{1} \in G$ we obtain a linear relation $\delta^{x_{i}}$ such that $x_{i+1}^{\prime}$ appears in $\delta^{x_{i}}$. Eliminating $g_{1}$ from each such pair we obtain a set

$$
C_{1}=\left\{\delta^{x_{1}}, \delta^{x_{3}}, \cdots, \delta^{x_{2} n-1}\right\}
$$

of $2^{n-1}$ linear relations in which $g_{1}$ does not appear and such that for each odd $i, 1 \leqq i<2^{n}$, there exists an $x_{i}^{\prime \prime} \in X$ with $x_{i}^{\prime \prime}$ appearing in $\delta^{x_{i}}$ but not in $\delta^{x_{k}}$ for $k<i$. Continuing in this manner to eliminate one element of $G$ at a time we obtain $C_{n}$ which consists of one nontrivial linear relation in which only elements of $X$ appear. This contradicts the linear independence of $X$ and completes the proof.

CoRollary 1.4. If $A$ positively spans an infinite-dimensional $L, B \subset A$, and card $B<\operatorname{card} A$, then $A \sim B$ is linearly dependent.

Proof. If $A \sim B$ is linearly independent then since card $(A \sim B)=$ card $A$ the theorem states that $\operatorname{pos}[(A \sim B) \cup B](=L)$ is not a subspace.

Corollary 1.5. If $L$ is infinite-dimensional and $A$ positively spans $L$ then there exists a $B \subset A$ such that card $B \geqq \operatorname{dim} L$ and $A \sim B$ linearly spans $L$ (in fact, is a linear basis for $L$ ).

Proof. Let $C \subset A$ be a linear basis for $L$. Then if card $(A \sim C)<$ card $C$ it follows by the theorem that pos $(A \sim C) \cup C(=L)$ is not a subspace. The contradiction implies card $(A \sim C) \geqq \operatorname{dim} L$, so we may take $B=A \sim C$.

Even if $B$ consists of a single element, Corollary 1.4 cannot be strengthened to state that there exists a nontrivial positive relation over $A \sim B$. The columns of the following array positively span a countably infinite-dimensional linear space. However, the first column must clearly appear in every nontrivial positive relation over $A$ :

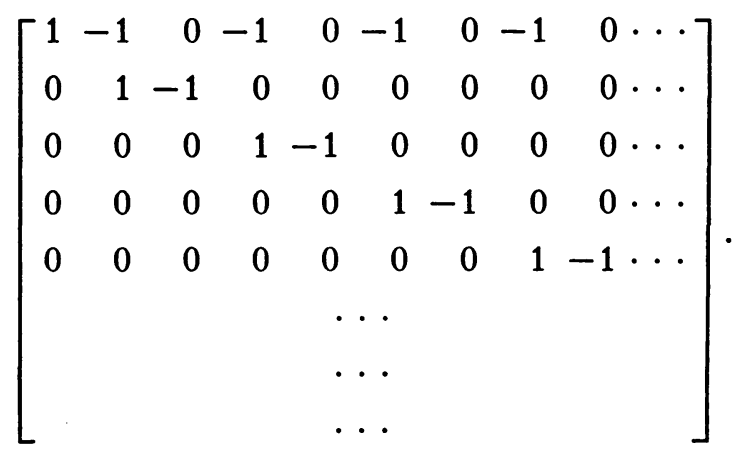


1.3. Relations among positively spanning sets, semispaces and open halfspaces. We will need the following definitions:

A variety $V$ in $L$ is any translate of a linear subspace of $L$. A hyperplane $H$ in $V$ is a set which is maximal among the varieties properly contained in $V$. For points $x$ and $y$ of $L$ we will use the notation $[x, y]=\{t x+(1-t) y \mid 0 \leqq t \leqq 1\}$. Then a subset $C$ of $L$ is convex if and only if for arbitrary $x \in C$ and $y \in C$, $[x, y] \subset C$. A subset $K$ of $L$ is a cone at a point $p \in L$ if and only if $\lambda(k-p)$ $\in\{k-p \mid k \in K\}$ for all $\lambda>0, k \in K$. If $H$ is a hyperplane in $V$ then a maximal convex subset $T$ of $V \sim H$ is an open halfspace bounded by $H$. If $p \in H$ then the open half-space $T$ is actually a convex cone at $p$ and we shall say that $T$ is an open halfspace at $p$. If $p \in L$, a semispace at $p$ is a maximal convex subset of $L \sim\{p\}$. The concept of semispace was introduced by Hammer [3] and investigated further by Klee [4]. We shall use some of the more elementary properties of semispaces found in these papers.

For a subset $A$ of $L$ consider the following assertions:

(1) $A$ positively spans $L$.

(2) $A$ intersects every open halfspace at $\varnothing$.

(3) $A$ intersects every semispace at $\varnothing$.

The following theorem collects the implications that exist among these properties.

THEOREM 1.6. If $L$ is finite-dimensional, (1) is equivalent to (2), and (2) implies (3) but not conversely. If $L$ is infinite-dimensional then the only valid assertions, in general, are that (1) implies (2) and (3). Even (2) and (3) together do not imply (1).

Proof. (1) implies (2) in general, for if $T$ is an open halfspace at $\varnothing$ then $L \sim T$ is a convex cone at $\varnothing$. Hence, if $A \subset L \sim T$ then every positive combination of $A$ is contained in $L \sim T$ and $A$ cannot span $L$.

That (2) implies (3) follows in the finite-dimensional case since a semispace at $\varnothing$ is then necessarily of the form $T \cup Q$ for some open halfspace $T$ at $\varnothing$ in $L$ and $Q$ some semispace at $\varnothing$ in the corresponding hyperplane.

(3) does not imply (2) since if $x \in L \sim\{\varnothing\}$ then the set $\{x,-x\}$ intersects every semispace at $\varnothing$.

To see that (2) implies (1) for finite-dimensional $L$, note that pos $A$ is a convex cone at $\varnothing$. Hence, if pos $A \neq L$ there exists a hyperplane of support at $\varnothing$, contrary to (2).

The following example shows that, in general, (2) does not imply either (1) or (3):

Let $L$ be an infinite-dimensional linear space. Let $B$ be a linear basis for $L$ which is well-ordered and has no last element. Let $C$ be the set of all $x \in L$ for which the last nonzero coefficient in the linear representation of $x$ in terms of $B$ is positive. Now we will show that $C$ intersects every open halfspace $T$ at $\varnothing$. Pick a point $x \in T$ and let $H$ be the hyperplane determined by 
$T$. Suppose $x=\sum_{b \in B} \lambda_{b}^{x_{b}}$ is the linear representation of $x$ and let $b_{1}$ be the last element of $B$ such that $\lambda_{b_{1}}^{x} \neq 0$. Since $H$ is infinite-dimensional there exists $y \in H$ such that if $y=\sum_{b \in B} \lambda_{b}^{y_{b}}$ is the linear representation of $y$ and $b_{0}$ is the last element of $B$ such that $\lambda_{b_{0}}^{\nu} \neq 0$ then $b_{0}>b_{1}$. We may assume $\lambda_{b_{0}}^{v}>0$ since otherwise we pick $-y \in H$. Then the point $x+y$ is in both $T$ and $C$.

Since pos $C=C \cup \varnothing, C$ does not positively span $L$.

Since $-C$ is a semispace at $\varnothing$ and $C \cap-C=\Lambda, C$ does not intersect every semispace at $\varnothing$.

If we let $C^{\prime}=C \cup\left\{-c_{0}\right\}$ where $c_{0} \in C$ then $C^{\prime}$ intersects every semispace at $\varnothing$. Hence, (2) and (3) together do not imply (1).

Finally, to see that (1) implies (3) in general, let $\lambda$ be a positive relation over $A$ and consider an arbitrary semispace $S$ at $\varnothing$. Then if no $a \in A$ which appears in the relation $\lambda$ is in $S$, all such $a$ are in $-S$. But then $\sum_{a \in A} \lambda_{a} a=\varnothing$ is in $-S$ contradicting the assumption that $S$ is a semispace.

1.4. Geometric characterizations of positively spanning sets. In this section we show that there are geometric conditions (of a nature similar to those investigated in Theorem 1.6) which are actually equivalent to the positively spanning property. One of these conditions will be described in terms of complementary cones, where we define a complementary cone $C$ to be a nonempty convex cone at $\varnothing$ whose complement $L \sim C$ is also a (possibly empty) convex cone at $\varnothing$. Another condition makes use of cones over semispaces, where we define a cone over a semispace to be a convex cone at $\varnothing$ consisting of the elements $\{t s \mid t>0, s \in S\}$, where $S$ is a semispace at an arbitrary point of $L$.

THEOREM 1.7. Let $A$ be a subset of the linear space $L$. Then the following are equivalent:

(1) A positively spans $L$.

(2) A intersects every complementary cone $C$.

(3) A intersects every cone over a semispace.

Proof. (1) implies (2), for if $A$ did not intersect the complementary cone $C$, then $A$, and consequently pos $A$, are contained in $L \sim C$. This contradicts the hypothesis that $A$ positively spans $L$.

For (2) implies (3), let $C$ be a cone over a semispace $S$. Then we need only show that $C$ is a complementary cone. Let $C=\{t s \mid s \in S, t>0\}$. Then clearly $L \sim C$ is a cone at $\varnothing$. To see that $L \sim C$ is convex, let $x_{1}$ and $x_{2}$ be in $L \sim C$ and suppose there exists an $r>0$, an $s \in S$ and $t, 0<t<1$, such that $t x_{1}+(1-t) x_{2}$ $=r s$. But then

$$
s=t\left(\frac{x_{1}}{r}\right)+(1-t)\left(\frac{x_{2}}{r}\right) \in L \sim S
$$

since $L \sim S$ is a convex set containing all positive multiples of $x_{1}$ and $x_{2}$. Hence, we have a contradiction. 
Finally, assuming (3) we wish to show that pos $A=L$. If pos $A \neq L$ then there exists $x \in L \sim\{\varnothing\}$ such that $x \notin \operatorname{pos} A$. Let $D=\{t(c-x) \mid t>0, c \in \operatorname{pos} A\}$. Then $D$ is a convex cone at $\varnothing$ which does not meet the cone $E=\{t x \mid t>0\}$ and neither cone contains $\varnothing$. Now by Corollary 1 of Hammer [3] there exist disjoint semispaces $S_{1}$ and $S_{2}$ at $\varnothing$ such that $S_{1} \supset D$ and $S_{2} \supset E$. Let $F=\left\{t\left(s_{2}+x\right) \mid t>0, s_{2} \in S_{2}\right\}$. Then $F$ is a cone over the semispace $S_{2}+x$ and $F \cap A=\Lambda$. For otherwise there exists a point $a \in A$ such that $a=t\left(s_{2}+x\right)$ for some $s_{2} \in S_{2}$ and $t>0$. But then $s_{2}=a / t-x \in \operatorname{pos} A-x \subset D \subset S_{1}$. This contradicts the disjointness of $S_{1}$ and $S_{2}$.

Observe that condition (3) above may not be replaced with " $A$ intersects every cone at $\varnothing$ over an open halfspace" since a cone at $\varnothing$ over an open halfspace is either another open halfspace or else is all of $L$.

\section{Positive Bases}

2.1. Introduction. We define a set $A \subset L$ to be positively dependent if and only if $a \in \operatorname{pos}(A \sim\{a\})$ for some $a \in A$. Then a set $A \subset L$ is positively independent if and only if it is not positively dependent. A set $B C L$ is a positive basis for $L$ if and only if $B$ positively spans $L$ and $B$ is positively independent.

Every linear space $L$ admits a positive basis, for if $B$ is a linear basis for $L$ then $A=B \cup\{-B\}$ is a positive basis for $L$. We shall call such a basis maximal. We shall be primarily concerned with positive bases which are not maximal.

The definition of positive dependence above is not equivalent to the statement that there exists a nontrivial positive relation $\lambda$ over $A$.

Clearly, any subset of a positively independent set of vectors is positively independent. However, a subset of a positive basis need not be a positive basis for any linear subspace since the subset need not positively span the subspace which it linearly spans.

In this section we consider some of the elementary properties of positive bases. Proofs of most of the theorems are omitted since they are easy extensions of those for the finite-dimensional case which appear in [2].

2.2. Obtaining positive bases as subsets of positively spanning sets. Examples show that there exists a set which positively spans an infinite-dimensional $L$ but which does not contain a positive basis for $L$. However, if $L$ is finite-dimensional we have:

Theorem 2.1. Suppose $L$ is a finite-dimensional linear space and $A$ positively spans $L$. Then $A$ contains a subset $B$ which is a positive basis for $L$.

Proof. Use Theorem 1.1 and familiar methods.

For an example of the type mentioned above, let $L$ be the space consisting of all sequences of real numbers which have only a finite number of terms different from zero. If $a$ is an element of $L$ denote by $a_{i}$ the $i$ th term of the sequence $a$. Now let $A$ consist of each element $a \in L$ for which there exists an integer $i$ such that $a_{j}=1$ for $j \leqq i$ and $a_{j}=0$ for $j>i$. Let $B$ consist of every 
element $a \in L$ for which there exists an integer $i$ such that $a_{j}=-\delta_{i j}$ for all $j$. Then $A \cup B$ positively spans $L$ but contains no subset which is a positive basis for $L$.

2.3. Spanned subspaces and minimal subspaces. We now define the object which plays a fundamental role in the structure of an arbitrary positive basis.

$A$ is a minimal positive basis for $L$ if and only if there exists an $a \in A$ such that $A \sim\{a\}$ is a linear basis for $L$. It follows from Corollary 1.4 that if $L$ has a minimal positive basis then $L$ is finite-dimensional. Hence, in light of Corollary 1.2 the choice of $a$ in the above definition is immaterial.

Every finite-dimensional $L$ has a minimal positive basis because if $B$ is a linear basis for $L$ then $B \cup\left\{\sum_{b \in B}(-b)\right\}$ is a minimal positive basis for $L$.

As we observed earlier, a subset of a positive basis is not, in general, a positive basis for a linear subspace. Hence, we make the definition: If $A$ is a positive basis for $L$ then a linear subspace $S \subset L$ is a spanned subspace with respect to $A$ if and only if $A \cap S$ is a positive basis for $S$. If, moreover, $A \cap S$ is a minimal positive basis for $S$ then $S$ is a minimal subspace with respect to $A$.

Since if $S$ is a minimal subspace then a proper subset of $A \cap S$ is linearly independent, it follows that one minimal subspace cannot contain another. The following theorems show that the minimal positive bases are involved in the structure of an arbitrary positive basis.

Theorem 2.2. If $A$ is a positive basis for a linear space $L$ and $a \in A$, then there exists a subset $M$ of $A$ which contains a and is a minimal positive basis for a linear subspace of $L$, i.e., pos $M$ is a minimal subspace containing $a$.

Proof. See Theorems 3.8 and 4.1 of [2].

Corollary 2.3. For a positive basis $A$ of a linear space $L$, any spanned subspace is a linear sum of minimal subspaces, and conversely.

Theorem 2.4. Let $A$ be a positive basis for the linear space $L$. Then if $S$ is a spanned subspace spanned by a finite set $B \subset A$ the following are equivalent:

(1) $S$ is a minimal subspace.

(2) The positive relation which involves the elements of $B$ and no others is unique (up to multiplication by a positive constant).

Proof. See Theorem 4.3 of [2].

As a consequence of the above theorem we may refer to the positive relation associated with a minimal subspace. We shall also call such a relation a minimal relation over $A$.

The following theorem gives another interesting characterization of the minimal positive bases.

THEOREM 2.5. If $A$ is a positive basis for a linear space $L$ then the following are equivalent:

(1) $A$ is a minimal positive basis. 
(2) For each $a \in A$, there exists an order on $L$ making $L$ an ordered vector space and such that the elements $A \sim\{a\}$ are all positive.

Proof. Assuming (1), $A \sim\{a\}$ is linearly independent and so $\operatorname{pos}(A \sim\{a\})$ does not contain $\varnothing$. Hence, by Proposition 13, $\$ 1$, Chapter II of Bourbaki [1], (2) holds.

Conversely, if $A$ is not a minimal positive basis there exists by Corollary 2.3 a positive relation $\lambda$ over $A$ which does not involve some $a \in A$. But then $\operatorname{pos}(A \sim\{a\})$ contains $\varnothing$ and there cannot exist an order on $L$ such that $L$ is an ordered vector space and all elements of $A \sim\{a\}$ are positive.

The linear relations over a positive basis $A$ are determined by the positive relations associated with the minimal subspaces of $A$ in the following manner.

TheOREM 2.6. Let $A$ be a positive basis for the linear space $L$. Then every positive (linear) relation over $A$ is a positive (linear) combination of the positive relations associated with the minimal subspaces of $L$ with respect to $A$.

Proof. See Theorem 4.4 of [2]. A direct proof based on the preceding results of this section is also possible.

\section{A Method of Characterizing Positive Bases}

3.1. Introduction. We will prove a theorem which characterizes, among the subsets $A$ of a linear space $L$ which linearly span $L$, those subsets which are positive bases for $L$. This theorem and the related results should be compared with Theorem 5.3 et seq. of [2].

It will be convenient to make use of the following rather trivial lemmas.

Lemмa 3.1. Let $A$ be a positive basis for a linear space L. If $M$ is a linearly independent set of minimal relations over $A$ and $M(A)=\{a \mid a$ appears in $m$ for some $m \in M\}$, then card $M \leqq$ card $M(A)$.

Lemma 3.2. Let $\langle$,$\rangle denote the inner product in Euclidean space E^{m}$. Let $\left\{a_{1}, a_{2}, \cdots, a_{n}\right\}$ be a set of nonzero vectors in $E^{m}$. Then $a_{1} \in \operatorname{pos}\left\{a_{2}, a_{3}, \cdots, a_{m}\right\}$ if and only if for each $b \in E^{m}$ such that $\langle b, a\rangle>0$ there exists an $i, 2 \leqq i \leqq n$, such that $\left\langle b, a_{i}\right\rangle>0$.

3.2. The characterization theorem. We are now ready for the main theorem of this section.

THEOREM 3.3. Let $A$ be a set of vectors which linearly span a linear space $L$. Then $A$ is a positive basis for $L$ if and only if there exists an index set $S$ and a non-negative real valued function $F$ defined on $A \times S$ with the following properties:

(1) For each $s \in S, F(a, s)=0$ for all but finitely many $a \in A$ and there exists $a b \in A$ such that $F(b, s) \neq 0$.

(2) For each $a \in A$, there exists an $s \in S$ such that $F(a, s) \neq 0$.

(3) $\sum_{a \in A} F(a, s) a=\varnothing$ for each $s \in S$, and if $\lambda$ is a linear relation over $A$ 
then there exist $\left\{s_{1}, s_{2}, \cdots, s_{n}\right\} \subset S$ and real numbers $r_{1}, r_{2}, \cdots, r_{n}$ such that $\lambda_{a}=r_{1} F\left(a, s_{1}\right)+r_{2} F\left(a, s_{2}\right)+\cdots+r_{n} F\left(a, s_{n}\right)$ for all $a \in A$.

(4) Let $\left\{s_{1}, s_{2}, \cdots, s_{n}\right\}$ be a finite subset of $S$ and define the set $B=\{a \mid a \in A$ and $F\left(a, s_{i}\right) \neq 0$ for some $\left.i, 1 \leqq i \leqq n\right\}$.

$B y$ (1) $B$ must be a finite set, say with $m$ elements. Then $B$ may be ordered, $B=\left\{b, b_{2}, \cdots, b_{m}\right\}$, in such a manner that there exists a positive integer $k \leqq m / 2$ satisfying the following conditions:

(i) For each $i \leqq k$ there exists a $t_{i}>0$ such that $F\left(b_{i}, s_{j}\right)=t_{i} F\left(b_{i+k}, s_{j}\right)$ for all $j, 1 \leqq j \leqq n$.

(ii) For each $i, 2 k<i \leqq m$, there exist non-negative real numbers $r_{1}, r_{2}, \cdots$, $r_{k}$ such that $F\left(b_{i}, s_{j}\right)=\sum_{\rho=1}^{k} r_{\rho} F\left(b_{\rho}, s_{j}\right)$ for all $j, 1 \leqq j \leqq n$.

Proof. Suppose $A$ is a positive basis for $L$. Let $M$ be a maximal linearly independent set of minimal relations over $A$. Then clearly, $M(A)=A$ where $M(A)$ is the set defined in Lemma 3.1. Now using Lemma 3.1 we may define a map $T$ of $A$ onto $M$. We will denote $T(a)$ by $\lambda^{a}$. Now let $S=A$ and define the function $F$ on $A \times S$ by $F(a, s)=\lambda_{a}^{s}$ for each $(a, s) \in A \times S$.

Then (1) follows since for each $s \in S, F(a, s)=\lambda_{a}^{s}$, and the positive relation $\lambda^{\circ}$ has the necessary properties by definition. Since $T$ maps $A$ onto $M$ and $M(A)=A,(2)$ is obvious. For (3) we need only apply Theorem 2.6.

To establish (4) let $\left\{s_{1}, s_{2}, \cdots, s_{n}\right\}$ be a finite subset of $S$ and consider the set $\left\{v_{b_{1}}, v_{b_{2}}, \cdots, v_{b_{m}}\right\}$ of $m$ vectors in $E^{n}$ defined by

$$
v_{b}=\left(\lambda_{b}^{s_{1}}, \lambda_{b}^{s_{2}}, \cdots, \lambda_{b}^{s_{n}}\right) \quad \text { for each } b \in B .
$$

If the set $D=\left\{v_{b} \mid b \in B\right\}$ is not positively independent we may choose $v_{b_{m}}$ such that $v_{b_{m}} \in D \cap \operatorname{pos}\left(D \sim\left\{v_{b_{m}}\right\}\right)$. If $D \sim\left\{v_{b_{m}}\right\}$ is not positively independent we may choose $v_{b_{m-1}}$ such that $v_{b_{m-1}} \in D \cap \operatorname{pos}\left(D \sim\left\{v_{b_{m}}, v_{b_{m-1}}\right\}\right)$. Continuing in this way we obtain a positively independent subset of $D$, say $\left\{v_{b_{1}}, v_{b_{2}}, \cdots, v_{b_{k}}\right\}$ such that $D \subset \operatorname{pos}\left(v_{b_{1}}, v_{b_{2}}, \cdots, v_{b_{k}}\right)$. Now (4) follows if we show that for each $i, 1 \leqq i \leqq k$, there exists a $t_{i}>0$ and a $b \in B, b \neq b_{i}$, such that $v_{b_{i}}=t_{i} v_{b}$.

If this last assertion is false, then there exists a $j, 1 \leqq j \leqq k$ such that $v_{b_{j}} \neq t v_{b}$ for any $t>0$ and $b \neq b_{j}$. Suppose $v_{b_{j}} \in \operatorname{pos}\left\{v_{b} \mid b \neq b_{j}\right\}$. Then we have $v_{b_{j}}=\sum_{i=1}^{k} r_{i} v_{b_{i}}$ where each $r_{i} \geqq 0$ and $r_{i}>0$ for some $i \neq j$. If $r_{j} \geqq 1$ we have

$$
\varnothing=\sum_{i=1 ; i \neq j}^{k} r_{i} v_{b_{i}}+\left(r_{j}-1\right) v_{b_{j}}
$$

which is impossible. If $r_{j}<1$ we have a contradiction of the positive independence of $\left\{v_{b 1}, v_{b_{2}}, \cdots, v_{b_{k}}\right\}$. Hence $v_{b_{j}} \notin \operatorname{pos}\left\{v_{b} \mid b \neq b_{j}\right\}$. Now by Lemma 3.2 there exists a vector $w=\left(t_{1}, t_{2}, \cdots, t_{n}\right)$ in $E^{n}$ such that $\left\langle w, v_{b_{j}}\right\rangle>0$ and $\left\langle w, v_{b}\right\rangle<0$ for $b \neq b_{j}$, i.e., $\sum_{i=1}^{n} t_{i} \lambda_{b_{j}}^{s_{i}}>0$ and $\sum_{i=1}^{n} t_{i} \lambda_{b}^{s_{i}}<0$ for all $b \neq b_{j}$. Hence, $\varnothing=\sum_{i=1}^{n}\left(t_{i} \sum_{b \in B} \lambda_{b}^{s_{i}} b\right)=\sum_{b \in B}\left(\sum_{i=1}^{n} t_{i} \lambda_{b}^{s_{i}}\right) b$ and in this last expression only the coefficient of $b_{j}$ is positive so that $b_{j} \in \operatorname{pos}\left\{b \mid b \in B, b \neq b_{j}\right\}$. This contradicts the hypothesis that $A$ is a positive basis for $L$. 
Conversely, assuming the existence of the function $F$ with the stated properties we must show that $A$ is a positive basis for $L$. Using (1), (2), (3) and Theorem 1.1 we see that $A$ positively spans $L$.

If $A$ is not positively independent then there exists an $a_{0} \in A$ and $\beta \in \mathfrak{B}\left(A \sim\left\{a_{0}\right\}\right)$ such that $a_{0}=\sum_{a \in A} \beta_{a} a$. Define a linear relation $\lambda$ over $A$ by letting $\lambda_{a}=-\beta_{a}$ for $a \neq a_{0}$ and $\lambda_{a_{0}}=1$. Now by (3) there exist a set $\left\{s_{1}, s_{2}, \cdots, s_{n}\right\} \subset A$ and real numbers $r_{1}, r_{2}, \cdots, r_{n}$ such that

$\lambda_{a}=\sum_{i=1}^{n} r_{i} F\left(a, s_{i}\right)=\left\langle\left(r_{1}, r_{2}, \cdots, r_{n}\right),\left(F\left(a, s_{1}\right), \cdots, F\left(a, s_{n}\right)\right)\right\rangle \quad$ for all $a \in A$.

Thus, since $\lambda_{a_{0}}>0$ while $\lambda_{a} \leqq 0$ for all $a \neq a_{0}$ we may apply Lemma 3.2 to conclude that

$$
\left(F\left(a_{0}, s_{1}\right), \cdots, F\left(a_{0}, s_{n}\right)\right) \notin \operatorname{pos}\left\{\left(F\left(a, s_{1}\right), \cdots, F\left(a, s_{n}\right)\right) \mid a \neq a_{0}\right\} .
$$

This contradicts (4) and completes the proof.

If $A$ is a positive basis for $L$ and $F$ satisfies the conditions of the above theorem with respect to $A$ with index set $S=A$ then we shall say that $F$ is associated with $A$.

If $F$ is associated with $A$ and $q$ is any map of $A$ onto $A$ then it is clear that $G$ is also associated with $A$ where

$$
G(a, b)=F(a, q b) \quad \text { for each }(a, b) \in A \times A .
$$

If $F$ is associated with $A$ then the example at the end of $\$ 1.2$ shows that it need not be true that for each $a \in A, F(a, b)=0$ for some $b \in A$.

3.3. Projections onto hyperplanes. Let $A$ be a positive basis and $A^{\prime} \subset A$ a linear basis for a linear space $L$. Let $a_{0}$ be an arbitrary element of $A^{\prime}$. Then we define $\Pi$ to be the natural projection of $L$ onto the hyperplane $H$ spanned by $A^{\prime} \sim\left\{a_{0}\right\}$, i.e., for $x=\sum_{a \in A^{\prime}} \lambda_{a} a \in L$ where $\lambda \in R A^{\prime}, \Pi x=\sum_{a \in A^{\prime} \sim\left\{a_{0}\right\}} \lambda_{a} a$.

If $a$ and $b$ are distinct elements of $A \sim\left\{a_{0}\right\}$ then $\Pi_{a} \neq \Pi_{b}$ since otherwise $a-b=r a_{0}$ for some nonzero $r$ and we have a contradiction of the positive independence of $A$. This remark will make it clear that $G$ in the theorem below is well defined.

Since $\Pi$ is a linear map it follows from Theorem 1.1 that $\Pi\left(A \sim\left\{a_{0}\right\}\right)$ $=\left\{\Pi a \mid a \in A \sim\left\{a_{0}\right\}\right\}$ positively spans $H$. Even if $-a_{0} \notin A, \Pi\left(A \sim\left\{a_{0}\right\}\right)$ need not be positively independent. In fact, let $L=E^{8}, A=\{a, b, c, d, e\}$ and $A^{\prime}=\{a, b, c\}$ where $a=(1,0,0), b=(0,1,0), c=(0,0,1), d=(-1,-1,0)$ and $e=(0,-1,-1)$. Then with $a_{0}=a$ we have $\Pi d=\Pi e+\Pi c$. Whenever $\Pi\left(A \sim\left\{a_{0}\right\}\right)$ is positively independent we have the following theorem.

Theorem 3.4. Let $A$ be a positive basis and $A^{\prime} \subset A$ a linear basis for a linear space $L$. Let $a_{0} \in A^{\prime}$ and $I$ be the natural projection of $L$ on the hyperplane $H$ spanned by $A^{\prime} \sim\left\{a_{0}\right\}$. If $\Pi\left(A \sim\left\{a_{0}\right\}\right)$ is positively independent (and hence a positive basis for $H$ ) then there exists an $F$ associated with $A$ such that if $G$ is 
defined by $G(\Pi a, \Pi b)=F(a, b)$ for all $(a, b) \in\left(A \sim\left\{a_{0}\right\}\right) \times\left(A \sim\left\{a_{0}\right\}\right)$ then $G$ is associated with $\Pi\left(A \sim\left\{a_{0}\right\}\right)$.

Proof. Let $F^{\prime}$ be any function associated with $A$. Since $\sum_{a \in A} F^{\prime}(a, b) a$ $=\varnothing$ for each $b \in A$, we have $\Pi\left(\sum_{a \in A} F^{\prime}(a, b) a\right)=\sum_{a \in A \sim\left\{a_{0}\right\}} F^{\prime}(a, b) \Pi a=\varnothing$ for each $b \in A$. Thus for each $b \in A, \lambda^{b}$ defined by $\lambda_{\Pi a}^{b}=F^{\prime}(a, b)$ is a positive relation over $\Pi\left(A \sim\left\{a_{0}\right\}\right)$. If $A$ is finite (and $n=$ card $A$ ) the set $\left\{\lambda^{b} \mid b \in A\right\}$ may be considered as a set of $n$ vectors in $E^{n-1}$. Hence, there exists a $b_{0} \in A$ such that $\lambda^{b_{0}} \in \operatorname{lin}\left\{\lambda^{b} \mid b \in A \sim\left\{b_{0}\right\}\right\}$. Define a map $q$ of $A$ onto $A$ by $q a_{0}=b_{0}$, $q b_{0}=a_{0}$ and $q a=a$ for $a \notin\left\{a_{0}, b_{0}\right\}$. If $F$ is defined by $F(a, b)=F^{\prime}(a, q b)$ for all $(a, b) \in A \times A$ then as we observed in the last section $F$ is also associated with $A$. If $A$ is infinite then there exists a map $p$ of $A \sim\left\{a_{0}\right\}$ onto $A$. Define $p^{*}$ mapping $A$ onto $A$ by $p^{*} a=p a$ for $a \in A \sim\left\{a_{0}\right\}$ and $p^{*} a_{0}=a_{0}$. Then if $F$ is defined by $F(a, b)=F^{\prime}\left(a, p^{*} b\right)$ for all $(a, b) \in A \times A, F$ is associated with $A$ as above.

Now with $G(\Pi a, \Pi b)=F(a, b)$ for all $(a, b) \in\left(A \sim\left\{a_{0}\right\}\right) \times\left(A \sim\left\{a_{0}\right\}\right)$, (1) of Theorem 3.3 follows from the observation that if there is a $b \in A \sim\left\{a_{0}\right\}$ such that $G(\Pi a, \Pi b)=0$ for all $a \in A \sim\left\{a_{0}\right\}$ then $F\left(a_{0}, b\right) b_{0}=\varnothing$ with $F\left(a_{0}, b\right)$ $\neq 0$, a contradiction.

Property (2) is taken care of by our choice of $F$.

For (3) let $\lambda$ be any linear relation over $\Pi\left(A \sim\left\{a_{0}\right\}\right)$. Then $\sum_{a \in A \sim\left\{a_{0}\right\}} \lambda_{\Pi a} \Pi a$ $=\varnothing$ or $\Pi\left(\sum_{a \in A \sim\left\{a_{0}\right\}} \lambda_{\Pi_{a} a} a\right)=\varnothing$ which implies $\sum_{a \in A \sim\left\{a_{0}\right\}} \lambda_{\Pi_{a} a} a=r a_{0}$ for some real number $r$. If we define $\beta$ by $\beta_{a}=\lambda_{\Pi a}$ for $a \in A \sim\left\{a_{0}\right\}$ and $\beta_{a_{0}}=-r$ then $\beta$ is a linear relation over $A$ so that applying (3) of Theorem 3.3 to $F$ and $\beta$ we know there exist $\left\{b_{1}, b_{2}, \cdots, b_{n}\right\} \subset A$ and real numbers $r_{1}, r_{2}, \cdots, r_{n}$ such that $\beta_{a}=\sum_{i=1}^{n} r_{i} F\left(a, b_{i}\right)$ for all $a \in A$. By our choice of $F$ we may assume $\left\{b_{1}, b_{2}, \cdots, b_{n}\right\} \subset A \sim\left\{a_{0}\right\}$. Then, $\lambda_{\Pi a}=\sum_{i=1}^{n} r_{i} G\left(\Pi a, \Pi b_{i}\right)$ for all $a \in A$ $\sim\left\{a_{0}\right\}$.

(4) follows easily from the fact that $F$ satisfies this property with respect to $A$.

Hence, $G$ satisfies all the properties required to be associated with $\Pi\left(A \sim\left\{a_{0}\right\}\right)$.

3.4. Direct sums. Let $A_{i}$ be a positive basis for $L_{i}, i \in\{1,2\}$, and suppose that $L$ is the direct sum of $L_{1}$ and $L_{2}$. Then it is easy to see that $A_{1} \cup A_{2}$ is a positive basis for $L$. The following theorem relates this situation to the associated functions.

THEOREM 3.5. Let $A$ be a positive basis for the linear space $L$. Then $L$ is the direct sum of spanned subspaces $L_{1}$ and $L_{2}$ if and only if there exist disjoint subsets $A_{1}$ and $A_{2}$ of $A$ and a function $F$ associated with $A$ such that $A_{1} \cup A_{2}=A$ and $F(a, b)=0$ if $a \in A_{i}$ and $b \notin A_{i}, i \in\{1,2\}$. Interchanging $a$ pair of subscripts if necessary, it then follows that $A_{i}$ is a positive basis for $L_{i}$ and that $F$ restricted to $A_{i} \times A_{i}$ is associated with $A_{i}$.

Proof. For the "if" part, using Theorem 2.6 and (3) of Theorem 3.3 we 
see that a minimal relation of $L$ with respect to $A$ involves only elements of one $A_{i}, i \in\{1,2\}$. Hence, $A_{i}$ is a positive basis for the space it linearly spans. Let $L_{i}=\operatorname{pos} A_{i}$. It remains to show $L_{1} \cap L_{2}=\Lambda$. If not, there exists an $x \neq \varnothing$ such that $x \in \operatorname{pos} A_{1}$ and $x \in \operatorname{pos} A_{2}$. But then $-x \in \operatorname{pos} A_{2}$. Thus $x=\sum_{a \in A 1} \lambda_{a} a$ for some $\lambda \in \mathfrak{B} A_{1},-x=\sum_{a \in A_{2}} \beta_{a} a$ for some $\beta \in \mathfrak{B} A_{2}$. Then $\varnothing=\sum_{a \in A} \alpha_{a} a$ where $\alpha_{a}=\lambda_{a}$ for $a \in A_{1}$ and $\alpha_{a}=\beta_{a}$ for $a \in A_{2}$ so that $\alpha \in \mathfrak{B} A$. Hence, by (3) of Theorem 3.3 there exist $\left\{a_{1}, a_{2}, \cdots, a_{m}\right\} \subset A_{1}$, $\left\{a_{m+1}, \cdots, a_{n}\right\} \subset A_{2}$ and real numbers $r_{1}, r_{2}, \cdots, r_{n}$ such that $\alpha_{a}$ $=\sum_{i=1}^{n} r_{i} F\left(a, a_{i}\right)$ for all $a \in A$. Then

$$
x=\sum_{a \in A_{1}} \lambda_{a} a=\sum_{a \in A_{1}} \alpha_{a} a=\sum_{a \in A_{1}} a \sum_{i=1}^{m} r_{i} F\left(a, a_{i}\right)=\sum_{i=1}^{m} r_{i} \sum_{a \in A} F\left(a, a_{i}\right) a=\varnothing
$$

which is a contradiction.

Conversely, if $L$ is the direct sum of spanned subspaces $L_{1}$ and $L_{2}$ then $A_{1}=A \cap L_{1}$ and $A_{2}=A \cap L_{2}$ are positive bases for $L_{1}$ and $L_{2}$ respectively. Hence, by Theorem 3.3 there exists $F_{1}$ associated with $A_{1}$ and $F_{2}$ associated with $A_{2}$. Now define $F$ by $F(a, b)=F_{i}(a, b)$ if $(a, b) \in A_{i} \times A_{i}$ for $i \in\{1,2\}$ and $F(a, b)=0$ if $(a, b) \in A \times A$ but $(a, b) \notin A_{i} \times A_{i}$ for $i \in\{1,2\}$. It is easy to check that $F$ is associated with $A$.

\section{Specialized Positive Bases}

4.1. Introduction. If $B$ is a linear basis for a linear space $L$, then for each $x \in L$ there exists a unique element $\lambda \in R B$ such that $x=\sum_{b \in B} \lambda_{b} b$. This important property does not carry over to positive bases, since the members of a positive bases always admit a positive relation.

If $A$ is a positive basis for $L$ and $x \in L \sim\{\varnothing\}$ then we define a minimal representation of $x$ with respect to $A$ to be an element $\lambda^{x} \in \mathfrak{B} A$ such that $x=\sum_{a \in A} \lambda_{a}^{x} a$ and if also $x=\sum_{a \in A} \beta_{a} a$ for some $\beta \in \Re A$ then the number of elements of $A$ appearing in $\beta$ is at least as large as the nuinber appearing in $\lambda^{x}$. We define the length of an element $x \in L$ to be the number of elements of $A$ appearing in a minimal representation of $x$.

In general, even a minimal representation of an element need not be unique, as is seen by considering the example at the end of $\$ 1.2$. However, we do have this type of uniqueness for certain positive bases. These are studied in the next section.

4:2. Unique minimal representations. The following lemmas about minimal representations are easily proved.

Lemma 4.1. Let $A$ be a positive basis for a linear space $L$ and $\lambda^{x}$ a minimal representation of $x$ with respect to $A$. Then for each positive relation $\beta$ over $A$, there exists an element $a$ of $A$ such that a appears in $\beta$ but not in $\lambda^{x}$.

LEмma 4.2. Let $A$ be a positive basis for a linear space $L$ and $x \in L \sim\{\varnothing\}$. If $\lambda^{x}$ and $\beta^{x}$ are distinct minimal representations of $x$ with respect to $A$ then the 
set of elements of $A$ appearing in $\lambda^{x}$ is different from the set of elements of $A$ appearing in $\beta^{x}$.

The following theorem gives a sufficient condition for the minimal representation to be unique.

THEOREM 4.3. Let $A$ be a positive basis for a linear space $L$ and suppose that any two distinct minimal subspaces of $L$ with respect to $A$ have only $\varnothing$ in common. Then the minimal representation of each $x \in L \sim\{\varnothing\}$ is unique.

Proof. Suppose the minimal representation of some element $x$ in $L \sim\{\varnothing\}$ is not unique. Then there exist distinct minimal representations $\lambda^{x}$ and $\beta^{x}$ satisfying $\varnothing \neq x=\sum_{a \in A} \lambda_{a}^{x} a=\sum_{a \in A} \beta_{a}^{x} a$. If some members of $A$ appear in both $\lambda^{x}$ and $\beta^{x}$ we may transpose terms to obtain $\left(^{*}\right) \sum_{i=1}^{j} \alpha_{i} a_{i}=\sum_{i=1}^{m} \delta_{i} b_{i}$ where $\alpha_{i}>0$ and $\delta_{i}>0$ for all $i, j<n$ and $m<n$ where $n$ is the length of $x$, and also $\left\{a_{1}, a_{2}, \cdots, a_{j}\right\} \cap\left\{b_{1}, b_{2}, \cdots, b_{m}\right\}=\Lambda$. Also, by Lemma $4.2, j \geqq 1$ and $m \geqq 1$. Consider the minimal subspace containing $a_{1}$ and let $a_{2}, a_{3}, \cdots, a_{k}$, $1 \leqq k \leqq j$, be (after re-indexing if necessary) the other elements of $\left\{a_{1}, a_{2}, \cdots, a_{j}\right\}$ contained in this subspace. Let $\gamma$ be the minimal relation associated with this minimal subspace. Then we have

$$
\sum_{i=1}^{k}\left(r \gamma_{a_{i}}-\alpha_{i}\right) a_{i}+\sum_{a \in A ; a \notin\left\{a_{1}, \ldots, a_{k}\right\}} r \gamma_{a} a+\sum_{i=1}^{k} \alpha_{i} a_{i}=\varnothing
$$

for all real numbers $r$. If we choose $r=r_{0}>0$ properly then there exists an $i_{0}, 1 \leqq i_{0} \leqq k$, such that $r_{0} \gamma_{a_{i_{0}}}-\alpha_{i_{0}}=0$ and $r_{0} \gamma_{a_{i}}-\alpha_{i} \geqq 0$ for all $i, 1 \leqq i \leqq k$. Now if we add the expression

$$
\sum_{i=1}^{k}\left(r_{0} \gamma_{a_{i}}-\alpha_{i}\right) a_{i}+\sum_{a \in A ; a \notin\left\{a_{1}, \ldots, a_{k}\right\}} r_{0} \gamma_{a} a
$$

to both sides of $\left(^{*}\right)$ we obtain

$$
\sum_{i=k+1}^{j} \alpha_{i} a_{i}=\sum_{i=1}^{m} \delta_{i} b_{i}+\sum_{i=1 ; i \neq i_{0}}^{k}\left(r_{0} \gamma_{a_{i}}-\alpha_{i}\right) a_{i}+\sum_{a \in A ; a \notin\left\{a_{1}, \cdots, a_{k}\right\}} r_{0} \gamma_{a} a
$$

in which $a_{i_{0}}$ does not appear. Notice that $\left(^{* *}\right) \neq \varnothing$ since otherwise $\sum_{i=1}^{k} \alpha_{i} a_{i}$ $=\varnothing$ and $\left\{a_{1}, a_{2}, \cdots, a_{k}\right\}$ span a minimal subspace. Then since also each $a_{i}, 1 \leqq i \leqq k$, appears in $\lambda^{x}$ we have a contradiction of Lemma 4.1.

Transposing the left side of $\left({ }^{* *}\right)$ we obtain $\left({ }^{* * *}\right) \varnothing=\sum_{i-1}^{m} \delta_{i} b_{i}+\left({ }^{* *}\right)$ $-\sum_{i=k+1}^{j} \alpha_{i} a_{i}$. We may use $(* * * *)$ to define a linear relation $\lambda^{*}$ over $A$ in which $a_{i_{0}}$ does not appear. Since $\left(^{* *}\right) \neq \varnothing, \lambda^{*}$ involves an element $a^{*}$ of $A$ from the same minimal subspace to which $a_{i_{0}}$ belongs. But by Theorem 2.6, $\lambda^{*}$ is a linear combination of minimal relations. Now, by hypothesis, the minimal subspaces are disjoint so this implies that since $a^{*}$ appears in $\lambda^{*}$ so must $a_{i_{0}}$. This contradiction completes the proof.

The above theorem has a number of interesting consequences which we now state. 
Corollary 4.4. If $A$ is a minimal positive basis for a linear space $L$ then the minimal representation of each $x \in L \sim\{\varnothing\}$ is unique.

Corollary 4.5. If $A$ is a maximal positive basis for a linear space $L$ then the minimal representation of each $x \in L \sim\{\varnothing\}$ is unique. Hence, for every linear space $L$ there exists a positive basis providing unique minimal representations.

Corollary 4.6. Let $A$ be a positive basis for a linear space $L$ and let $F$ be associated with $A$ as in \$3. Consider the vector $(F(a, b)) \in R^{A}$ for a fixed $b \in A$ and let $E$ be the set of all such vectors. Let $G$ be a maximal positively independent subset of $E$. If for each $a_{0} \in A$ there exists a unique $b_{0} \in A$ such that $\left(F\left(a, b_{0}\right)\right) \in G$ and $F\left(a_{0}, b_{0}\right) \neq 0$ then the minimal representation of each $x \in L \sim\{\varnothing\}$ is unique.

Corollary 4.7. If $A$ is a positive basis for a linear space $L$ obtained by applying the method of Theorem 4.14 to a positive basis $B$ for which the minimal subspaces have only $\varnothing$ in common then the minimal representation of each $x \in L \sim\{\varnothing\}$ is unique.

4.3. Strong positive bases. If $A$ is a subset of a linear space $L$ we shall say that $A$ is strongly positively independent if and only if for each $\lambda \in \Re A$ and $\beta \in \Re A$, where $\lambda$ and $\beta$ are not positive relations over $A, \sum_{a \in A} \lambda_{a} a$ $=\sum_{a \in A} \beta_{a} a$ implies there exists an $a \in A$ which appears in both $\lambda$ and $\beta$. Notice that strong positive independence implies ordinary positive independence but not conversely as the theorem below shows. We define a strong positive basis $A$ for $L$ as a positive basis $A$ for $L$ such that $A$ is strongly positively independent. We now show that this new concept is actually equivalent to the property involving pairwise disjoint minimal subspaces which was discussed in the last section. The following lemma will be useful.

Lemma 4.8. Let $A$ be a positive basis for a linear space $L$. If each pair of minimal subspaces of $L$ with respect to $A$ have only $\varnothing$ in common then each pair of spanned subspaces with no basis element in common have only $\varnothing$ in common.

Proof. Let $S_{1}$ and $S_{2}$ be spanned subspaces with no basis element in common. Define $A_{i}=S_{i} \cap A$, $i \in\{1,2\}$. Then $A_{1} \cap A_{2}=\Lambda$. Define $F$ on $A \times A$ as in the proof of Theorem 3.3. Now apply Theorem 3.5.

Corollary 4.9. Let $A$ be a positive basis for a linear space $L$. Then $L$ is the direct sum of its minimal subspaces if and only if each pair of minimal subspaces have only $\varnothing$ in common.

TheOREM 4.10. Let $A$ be a positive basis for a linear space L. Then $A$ is a strong positive basis if and only if each pair of minimal subspaces of $L$ with respect to $A$ have only $\varnothing$ in common.

Proof. Suppose $A$ is a strong positive basis. If there exist minimal sub- 
spaces $M_{1}$ and $M_{2}$ which have an element $x \neq \varnothing$ in common then there exist $\lambda$ and $\beta$ in $\mathfrak{B} A$ such that every element of $A$ appearing in $\lambda$ is in $M_{1}$ and every element of $A$ appearing in $\beta$ is in $M_{2}$ where $\lambda$ and $\beta$ are not relations and satisfy $\left(^{*}\right) x=\sum_{a \in A} \lambda_{a} a=\sum_{a \in A} \beta_{a} a$. We may suppose that $\lambda$ and $\beta$ satisfy the property $(Z)$ that each involves an element not appearing in the other; otherwise, we may add the minimal relation $\alpha_{1}$ associated with $M_{1}$ to $\lambda$ and the minimal relation $\alpha_{2}$ associated with $M_{2}$ to $\beta$. By Theorem $2.4, \alpha_{1}$ and $\alpha_{2}$ satisfy property $(Z)$ and since $\lambda$ and $\beta$ involve only elements appearing in $\alpha_{1}$ and $\alpha_{2}$ respectively, we conclude that $\alpha_{1}+\lambda$ and $\alpha_{2}+\beta$ also satisfy property (Z). Now we may transpose like terms of $\left({ }^{*}\right)$ to obtain an equation $\left({ }^{*}\right) \sum_{a \in A} \lambda_{a}^{*} a$ $=\sum_{a \in A} \beta_{a}^{*} a$ where $\lambda^{*}$ and $\beta^{*}$ are in $\mathfrak{P} A$ and such that no element of $A$ appears in both $\lambda^{*}$ and $\beta^{*}$. Property (Z) assures us that both $\lambda^{*}$ and $\beta^{*}$ involve some (but not the same) element of $A$. If $\lambda^{*}$ or $\beta^{*}$ is a relation then both are relations and since every element of $A$ appearing in $\lambda^{*}\left(\beta^{*}\right)$ must also appear in $\lambda(\beta)$ we have every element of $M_{1} \cap A\left(M_{2} \cap A\right)$ appearing in $\lambda^{*}\left(\beta^{*}\right)$. Hence $M_{1} \cap A \cap M_{2}=\Lambda$ and $\lambda^{*}=\lambda\left(\beta^{*}=\beta\right)$. This contradicts the choice of $\lambda$ and $\beta$ and thus shows that $\lambda^{*}$ and $\beta^{*}$ are not relations. Hence, $\left({ }^{* *}\right)$ is a contradiction of the strong positive independence of $A$.

For the converse, let $\varnothing \neq x=\sum_{a \in A} \lambda_{a} a=\sum_{a \in A} \beta_{a} a$. Define $A_{1}=\{a \mid a$ appears in $\lambda\}, A_{2}=\{a \mid a$ appears in $\beta\}$ and $S_{i}=$ spanned subspace generated by $A_{i}, i \in\{1,2\}$. Then since $x \in S_{1} \cap S_{2}$, Lemma 4.8 implies the existence of an $a \in A$ such that $a \in S_{1} \cap S_{2}$.

Corollary 4.11. If $A$ is a strong positive basis for a linear space $L$ then the minimal representation of each $x \in L \sim\{\varnothing\}$ is unique.

It would be interesting to know if the converse of Theorem 4.3 and of the above corollary are true, i.e., if $A$ is a positive basis for which the minimal representations are unique, must $A$ be strong?

4.4. Change of basis. If $A$ and $B$ are linear bases for $L$ then, as is well known, we may find a biunique linear map of $L$ onto $L$ which carries $A$ onto $B$. There is no hope for such a theorem if $A$ and $B$ are merely positive bases. Indeed, if $L$ is finite-dimensional then card $A$ need not be equal to card $B$. However, if $A$ and $B$ are strong positive bases whose minimal subspaces correspond in a certain natural way then we may normalize $A$ and $B$, in a sense to be defined, to obtain $A^{*}$ and $B^{*}$ for which there exists a biunique map of $L$ onto itself carrying $A^{*}$ onto $B^{*}$.

If $A$ is a strong positive basis for $L$ we will denote by $M(A)$ a set of minimal relations of $L$ with respect to $A$ which includes exactly one relation involving each element of $A$. Clearly, if $M^{\prime}(A)$ is another such set then each element of $M^{\prime}(A)$ is obtained by multiplying an element of $M(A)$ by a positive real number. Now let $A$ and $B$ be strong positive bases for $L$. We shall say that $A$ and $B$ have the same pattern of minimal subspaces or the same $P M S$ if for some (and thus each) pair $M(A), M(B)$ there exists a biunique map $T$ 
of $M(A)$ onto $M(B)$ such that for each $\lambda \in M(A), \lambda$ and $T \lambda$ involve the same number of elements of $A$ and $B$ respectively. A positive basis $A$ is called a normal positive basis provided there exists a set $M(A)$ such that $\lambda \in M(A)$ implies $\lambda_{a}=1$ or $\lambda_{a}=0$. If $A$ is a strong positive basis for $L$ then we may define a normal positive basis $A^{*}$ as follows. Choose a set $M(A)$ and let $A^{*}=\left\{\lambda_{a} a \mid a \in A, \lambda \in M(A)\right.$ and $\left.\lambda_{a} \neq 0\right\}$. Clearly, $A$ and $A^{*}$ have the same PMS. We shall say that $A^{*}$ is a normalization of $A$. We are now ready to prove the result stated at the beginning of this section.

THEOREM 4.12. If $A$ and $B$ are strong positive bases for a linear space $L$ with the same PMS and $A^{*}$ and $B^{*}$ are normalizations of $A$ and $B$ respectively then there exists a biunique linear map $\Phi$ of $L$ onto $L$ such that $\Phi\left(A^{*}\right)=B^{*}$.

Proof. Since $A$ and $B$ have the same PMS so do $A^{*}$ and $B^{*}$. Let $M\left(A^{*}\right)$ and $M\left(B^{*}\right)$ have the property used in the definition of normal bases. We can find a biunique map $\Phi^{\prime}$ of $A^{*}$ onto $B^{*}$ such that if $\lambda \in M\left(A^{*}\right)$ and $C \subset A^{*}$ is the set of elements appearing in $\lambda$ then there exists $\beta \in M\left(B^{*}\right)$ such that $\Phi^{\prime} C$ is the set of elements appearing in $\beta$. For each $\lambda \in M\left(A^{*}\right) \Phi^{\prime}$ may be extended to a linear map of lin $C$ onto $\operatorname{lin}\left(\Phi^{\prime} C\right)$. Now use Corollary 4.9 to define $\Phi$ on $L$.

CoRollary 4.13. If $A$ and $B$ are normalized minimal positive bases for a linear space $L$ (i.e., $E^{n}$ ) then there exists a biunique linear map of $L$ onto itself carrying $A$ onto $B$.

4.5. Reducible bases. If a positive basis $A$ for $L$ has the property that there exist elements $a_{1}$ and $a_{2}$ in $A$ such that $\left(a_{1}+a_{2}\right) \cup\left(A \sim\left\{a_{1}, a_{2}\right\}\right)$ is also a positive basis for $L$ then we say that $A$ is reducible. We shall show that a positive basis is reducible if and only if it is not minimal.

Theorem 4.14. Let $A$ be a positive basis for a linear space L. Suppose $B=\left\{b_{1}, \cdots, b_{n}\right\} \subset A$ has the property that for each $i, 1 \leqq i \leqq n$, there exists a minimal subspace $M_{i}$ of $L$ with respect to $A$ such that $M_{i} \cap B=\left\{b_{i}\right\}$. Let $r_{1}, \cdots, r_{n}$ be positive real numbers. Then

$$
C=\left\{\sum_{i=1}^{n} r_{i} b_{i}\right\} \cup(A \sim B)
$$

is a positive basis for $L$.

Proof. If $C$ is not positively independent we have $\sum_{i=1}^{n} r_{i} b_{i}=\sum_{a \in A \sim B} \beta_{a} a$ for some $\beta \in \mathfrak{B}(A \sim B)$. Let $\lambda^{i}$ be the minimal relation associated with $M_{i}$. Then

$$
-r_{i} b_{i}=\frac{r_{i}}{\lambda_{b}^{i}} \sum_{a \in A \sim B} \lambda_{a}^{i} a
$$

$r_{1} b_{1}=\sum_{a \in A \sim B} \beta_{a} a+\sum_{i=2}^{n}\left(r_{i} / \lambda_{b}^{i}\right) \sum_{a \in A \sim B} \lambda_{a}^{i} a$. This is a contradiction of the 
positive independence of $A$.

To show that $C$ positively spans $L$ let $x$ be an arbitrary element of $L \sim\{\varnothing\}$. Then there is a $\lambda \in \mathfrak{B} A$ such that $x=\sum_{a \in A} \lambda_{a} a$. Now we have

$$
x=r_{0} \sum_{i=1}^{n} r_{i} b_{i}+\sum_{a \in A \sim B} \lambda_{a} a+\sum_{i=1}^{n}\left(\lambda_{b_{i}}-r_{0} r_{i}\right) b_{i}
$$

and we may choose $r_{0}$ so that $\lambda_{b_{i}}-r_{0} r_{i}<0$ for each $i, 1 \leqq i \leqq n$. This means we can represent $x$ by

$$
x=r_{0} \sum_{i=1}^{n} r_{i} b_{i}+\sum_{a \in A \sim B} \lambda_{a} a+\sum_{i=1}^{n} \frac{r_{0} r_{i}-\lambda_{b_{i}}}{\lambda_{b}^{i}} \sum_{a \in A \sim B} \lambda_{a}^{i} a
$$

which is the required form.

Corollary 4.15. A positive basis $A$ for a linear space $L$ is reducible if and only if $A$ is not minimal.

Proof. Clearly a minimal basis may not be reduced. For the converse we need only note that if $A$ is not minimal there exist at least two distinct minimal subspaces $M_{1}$ and $M_{2}$ and so by Theorem $2.4 M_{1}$ contains an element of $A$ not in $M_{2}$ and conversely.

It is not true that if $A$ is a positive basis for $L$ and $\left\{a_{1}, a_{2}\right\} \subset A$ is a linearly independent subset such that $A \sim\left\{a_{1}, a_{2}\right\}$ linearly spans $L$ then $C=\left\{a_{1}+a_{2}\right\}$ $\cup\left(A \sim\left\{a_{1}, a_{2}\right\}\right)$ must be a positive basis for $L$. Let $L=E^{8}$ and take the columns of the following matrix as the elements of $A$ and the last two columns as $a_{1}$ and $a_{2}$ respectively. Then the second column does not appear in any positive relation over $C$ :

$$
\left[\begin{array}{rrrrr}
1 & 1 & 1 & 1 & -2 \\
0 & 1 & -1 & 0 & 0 \\
1 & 0 & 0 & -1 & 0
\end{array}\right] .
$$

\section{REFERENCES}

1. N. Bourbaki, Espaces vectoriels topologiques, Actualités Sci. Ind. No. 1189, Hermann, Paris, 1953.

2. Chandler Davis, Theory of positive linear dependence, Amer. J. Math. 76 (1954), 733-746.

3. Preston C. Hammer, Maximal convex sets, Duke Math. J. 22 (1955), 103-106.

4. V. L. Klee, Jr., The structure of semispaces, Math. Scand. 4 (1956), 54-64.

UNIVERSITY OF WASHINGTON, Seattle, Washington

University of California, Riverside, California 